\title{
Simulations and pre-experiment uncertainty analyses for a hydrogen diffusion experiment using a "two side purged membrane" setup
}

\author{
Frederik Arbeiter, D. Klimenko, Ch. Klein, G. Schlindwein, V. Pasler, A. von der Weth
}

\section{Background and Objective}

An experiment "Q-PETE" representative for the situation in the Helium Cooled Pebble Bed breeding zone and suitable for validation of relevant tritium transport codes is planned. In a temperature controlled setup a hydrogen loaded feed gas is directed over a steel membrane into which it can permeate. On the other side of the membrane a sweep gas flow collects the permeated hydrogen and transports it to a gas analysis (QMS) for quantitative time resolved detection.

\section{$\rightarrow$ Methods to handle time resolved permeation experiments with sweep gas, considering the residence time distribution of the sweep gas are introduced.}

\section{$\underline{\text { Issue }}$}

- The time resolved permate flux signal over the membrane $j_{Q Q}^{M}(t)$ is of interest for code validation and material parameter (Diffusivity $D$, Sieverts constant $\mathrm{K}_{\mathrm{S}}$ ) determination

- The purge flow residence time distribution in the permeate chamber (PSC) and the piping effects a lag and a dispersion in the measurable concentration signal $x_{Q M S}(t)=\dot{j}_{Q Q}^{Q M S}(t) / j_{S G}$

$\rightarrow$ The difference between $j_{Q Q}^{M}(t)$ and $x_{Q M S}(t)$ must be described!

\section{Simulation methodology}

System response $j_{Q Q}^{Q M S}(t)=j_{Q Q}^{M}(t) * g(t)=\int_{u=0}^{t} s(u) \cdot g(t-u) d u$

- Impulse response function $\boldsymbol{g}(\boldsymbol{t})$ is residence time distribution

- Convolution integral $j_{Q Q}^{M}(t) * g(t)$ is numerically solved

Further model for QMS sampling and noise applied

$\rightarrow$ Complete system signal simulation (prediction) implemented .

\section{Uncertainty estimation}

- As figure of merit, propagated uncertainties to $\mathrm{D}$ and $\mathrm{K}_{\mathrm{S}}$ are estimated for a simplified analytic evaluation method.
(1) $D \approx 0.16877 \cdot w_{M}^{2} / \Delta \tau$,
(2) $\phi=D K_{S}=\frac{2 w_{M}}{A_{M}} \cdot \frac{x_{Q M S}(t \rightarrow \infty) \cdot j_{S G}}{\sqrt{p_{Q Q, 1}}-\sqrt{p_{Q Q, 2}}}$

- Experimental setpoints can be optimized. For example: large flow rate and/or large membrane thickness: $\rightarrow$ good time resolution but low signal level (and vice versa).

$\rightarrow$ The residence time distribution in the permeation setup volume before the analysis station has significant effect and can be predicted by appropriate methods.

$\rightarrow$ Experimental uncertainties of $\sim \mathbf{1 5} \%$ for the derived Sieverts constant for breeder zone typical conditions are expected for the Q-PETE purged permeation experiments.

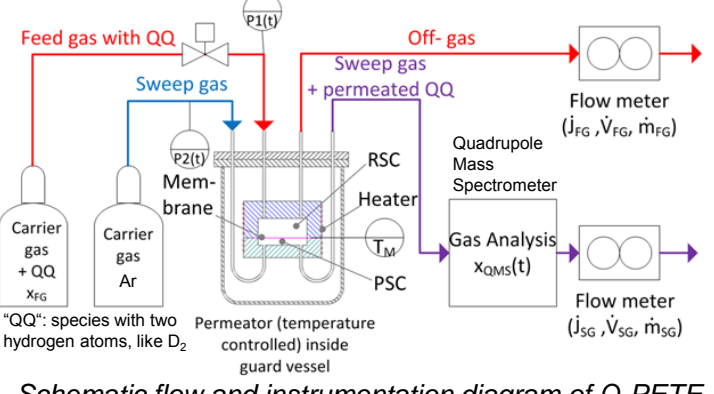

Schematic flow and instrumentation diagram of Q-PETE

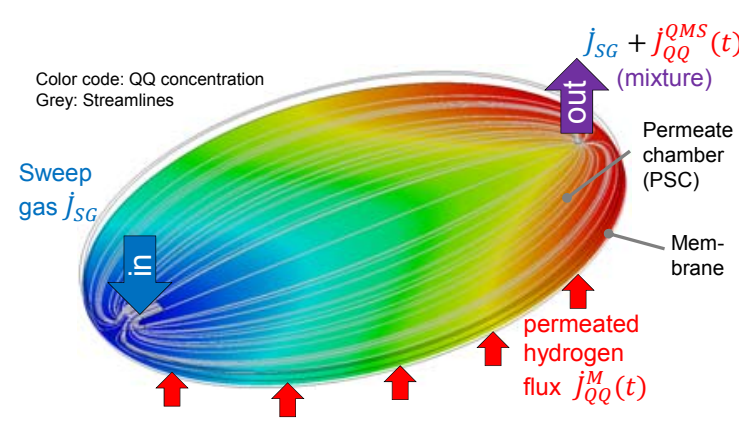

Flow and concentration field in the purged permeate chamber
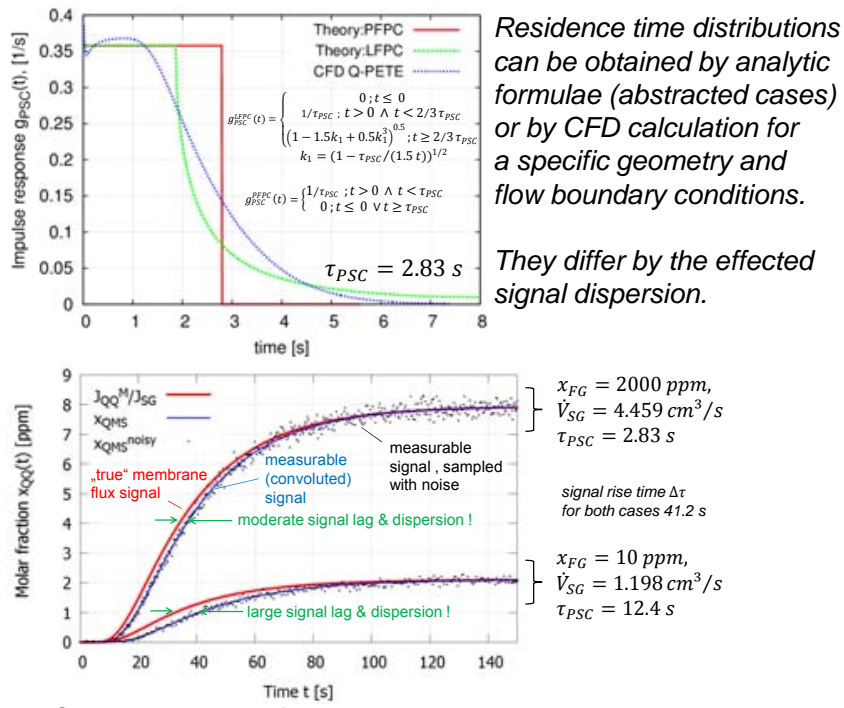

Simulated signals for two experimental conditions $x_{F G}$ : feed gas hydrogen content. $\dot{V}_{S G}$ : Sweep gas flow rate

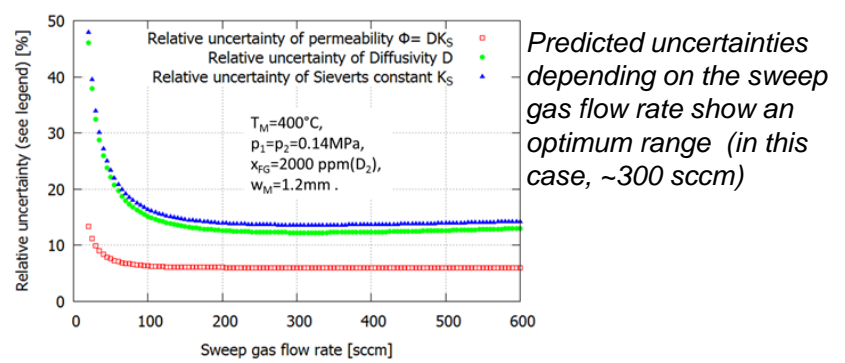

